\title{
Business Intelligence Solution for an SME: A Case Study
}

\author{
Raghavendra Raj, Shun Ha Sylvia Wong and Anthony J. Beaumont \\ School of Engineering and Applied Science, Aston University, Aston Triangle, Birmingham B4 7ET, U.K.
}

Keywords: $\quad$ Business Intelligence, Data Warehouse, Microsoft BI, SME.

Abstract: $\quad$ Business Intelligence (BI) leverages the usefulness of existing information. It equips business users with
relevant information to perform various analyses to make key business decisions. Over the last two decades,
BI has become a core strategy for the growth of many companies, in particular large corporations. However,
studies show that small and medium-sized enterprises (SMEs) lag behind in implementation and
exploitation of BI solutions. To stay ahead of the competition, SMEs must be able to monitor and
effectively use all of their resources, in particular information resources, to assist them in making important
business decisions. In this paper, we examine the challenges such as lack of technical expertise and limited
budget when implementing a BI solution within an SME in the UK. In light of our experiences in tackling
these issues, we discuss how these challenges can be overcome through applying various tools and
strategies and the potential benefits.

\section{INTRODUCTION}

In the last couple of decades, regardless of the size, companies have generated large volumes of data from internal and external environments. The ability to efficiently manage, access and analyse large, though seemingly unrelated, volumes of data is essential to business decision-making, otherwise potential business opportunities may be missed and important lessons may not be learnt. The rapid changes in business climate and conditions have brought Business Intelligence (BI) into the limelight even for small and medium-size enterprises (SMEs). To bring about a competitive advantage, companies have to think of new ways to improve their businesses. One among the several strategies has been the adoption of Data Warehouse (DW) and Business Intelligence (BI). This has become an essential component of contemporary business decision support systems. However, amongst SMEs, there is a general lack of technical expertise required to convert data into information to facilitate an informed business decision making process. Furthermore, the lack of understanding of the benefits of BI and budget also make SMEs reluctant to invest in adopting any BI solutions.

Business Intelligence in itself is not a novel concept. This term was first put forward by Luhn (1958). As Burstein and Holsapple (2008) recalled, this concept was reintroduced by Howard Dresner in
1989. Negash and Gray (2003) defined BI systems as systems which "combine data gathering, data storage, and knowledge management with analytical tools to present complex and competitive information to planners and decision-makers". Such systems typically utilise data from a centralised data repository that were brought together from different data sources. Business Intelligence has grown significantly since then due to the advancement of BI tools and also the readiness of companies to adopt new strategies to stay ahead of their game. Some of the technologies such as Decision Support Systems (DSS), Executive Information Systems (EIS), Data Warehouse (DW), Online Analytical Processing (OLAP) and Data Mining have had a significant impact on the increase in popularity of BI.

According to a survey of CIOs in 2012 (Gartner, 2012), BI was ranked as the top technology priority by the companies as BI enabled them to create new capabilities. Howson (2008) also noted that BI helped employees in all cadres to interact with, and analyse, business data in order to manage and increase the efficiency of the company, to identify any new opportunities and to facilitate operation reengineering. Watson and Wixom (2007) pointed out that information retrieval could be faster and cheaper by implementing BI solutions. They also mentioned that BI solutions could help organisations achieve their business goals. Chugh and Grandhi 
(2013) summarised key benefits of implementing BI within an enterprise, which include:

- Ability to analyse data from multiple sources and using various dimensions;

- Enabling managers to make intelligent business decisions by identifying important patterns in the business transaction data;

- Improved accuracy in predictions of business trend;

- Increased operational efficiency through identifying root causes of problems.

\subsection{BI Components}

To generate business intelligence, data from business transactions needs to undergo an ETL (Extract-Transform-Load) process which cleanses, transforms and restructures the data into a data warehouse or a data mart. Data warehouses and data marts are essentially databases specially designed for facilitating data analysis and knowledge discovery. The data analyses are guided by the Key Performance Indicators (KPIs) of the organisation and they vary from business to business. A data visualisation tool is used to present the results of data analyses to BI users. These results may appear in the form of standard reports based on regular queries or as on-demand reports which show information about business performance and efficiency. Figure 1 summarises the key components of a BI solution.

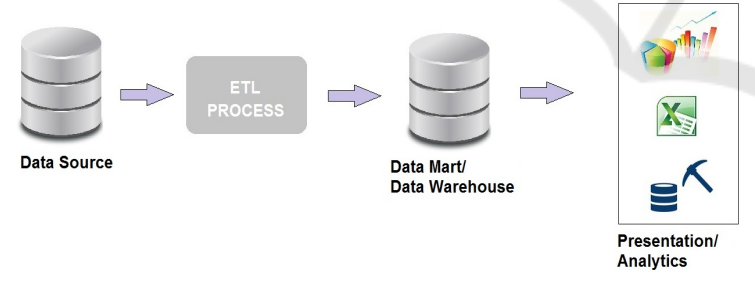

Figure 1: Key Components of BI.

\subsection{BI Vendors}

As mentioned earlier, BI solutions comprise multiple technological components. In the past, each BI component was built and delivered by specialised vendors. Selecting an appropriate combination of BI components to meet a business's need requires technical expertise which is often not readily available in a business setting. The need to integrate various $\mathrm{BI}$ components also pushes up the overall implementation cost of a BI solution. Nowadays, suppliers have developed BI components that could be easily integrated. This massively reduces the cost to implement BI. However, there remains the need to identify a suitable strategy to integrate BI components with a company's existing systems.

Some of the major players in the BI field are well-known names like Oracle, Microsoft, IBM, SAS and SAP. Over the last few years, some smaller vendors like Qlik and Tableau are gaining popularity. Amongst the major suppliers of $\mathrm{BI}$ solutions, Microsoft and IBM are two suppliers whose BI products cover the entire stack of BI components. Suppliers like Microsoft also deliver these BI components as a part of the Microsoft Developer Network (MSDN) license (Microsoft, 2016c) without any extra cost.

\subsection{BI Usage}

Existing business sectors which make use of BI to make some key business decisions include: banking, health service, IT companies, insurance companies, manufacturing industry, financial services and many more. As mentioned, the majority of large organisations are already drawing benefits from using BI and BI has become one of the major strategies to maintain a competitive edge.

A survey conducted by McCabe (2012) found that $33 \%$ of medium-size companies already used BI solutions and a further $28 \%$ planned to use BI solutions. However, amongst smaller organisations, only $16 \%$ were using $\mathrm{BI}$ solutions and $16 \%$ planned to use BI solutions. Though McCabe's (2012) survey showed that the adoption of BI amongst SMEs was slow, there was an upward trend in the awareness of the need for BI.

Studies (Horakova and Skalska, 2013) have shown that different departments and business units within a company use some kind of BI tools, and the major users of BI include managers from: sales, marketing, purchasing, accrual, finance, accounting, human resource and IT. It is therefore clear to see that $\mathrm{BI}$ is not just for top-level management such as Managing Directors. A wide range of business decision-making processes can also be benefited from using BI.

\subsection{BI Solutions}

Given the cost and complexity involved in most BI solutions, a large number of SMEs simply adopt a BI solution which utilises spreadsheets such as MS Excel integrated with a database (McCabe, 2012; Tutunea and Rus, 2012). However, as typical spreadsheet software does not come with a rich set of data analysis and visualisation tools to facilitate 
knowledge discovery, the resulting level of BI is rudimentary.

Another contemporary BI solution comes in a form of Software as a Service (SaaS). SaaS is essentially a software application deployed in the cloud. Rather than purchasing the software application from a vendor and installing it in the company's server, companies pay monthly or yearly subscriptions to gain access to the application via the Internet. With SaaS, company data is typically stored in the cloud and is managed by the service provider (Papazoglou, 2003). One typical application of BI supported by SaaS is to provide intelligence on customer satisfaction in order to bring about an improved Customer Relationship Management (CRM). BI as a Service could be very beneficial for SMEs because they are cost effective and also relatively easy to implement. The major concern of using a cloud-based service is data security, privacy and data ownership.

While data ownership and privacy are not issues for a locally-hosted BI solution, there is the need to consider how data visualisation reports can be shared with other stakeholders. BI using web technologies, e.g. Google Chart (Google, 2016), should enable web users to maintain reports in the web, distribute the reports to interested users, annotate the content and also generate ad-hoc reports from web applications dynamically.

\section{BI AND THE SME}

The European Commission (EC) defined a small and medium-size enterprise (SME) as a business which has less than 250 staff, with a turnover of no more than $€ 50 \mathrm{~m}$ and $99 \%$ of businesses in the EU fall in this category (European Commission, 2016). With a relatively small turnover, SMEs typically do not have additional financial and human resources to invest in new technologies such as BI systems that are not core to their businesses. Hence, it comes as no surprise that the adoption of BI amongst SMEs has been slow. Tutunea and Rus (2012) noted that cost-effective cloud-based solutions to BI exist and an efficient BI solution can give SMEs a competitive advantage. However, the issue of lacking expertise and technical know-how to select and adopt a suitable BI solution still poses a challenge for most SMEs.

For SMEs, the term Business Intelligence is often misperceived as a technology that is applicable to large organisations only. This misconception about BI means that most decisions made by the top level management in SMEs are based on the results from various Information and Communications Technology (ICT) tools built as a part of the company's infrastructure (Tutunea and Rus, 2012). There is a general opinion that SMEs are trailing behind in adopting Business Intelligence to assist in decision-making. There are a lot of studies and articles describing successful adoption and utilisation of BI amongst large organisations but similar successful stories amongst SMEs are uncommon (Fink and Ploder, 2009).

Irrespective of size and sector, companies appreciate the necessity for, and the benefits of, BI to assist in the business decision-making process. However, with the complexity involved in a typical $\mathrm{BI}$ implementation process, there is a high risk for the project to fail in SMEs. Hence, SMEs need a simple, flexible and efficient solution.

\subsection{Challenges of BI Adoption in SMEs}

There are various factors that affect SMEs' planning to adopt BI solutions:

- BI solutions are often expensive, for example, a cloud-based BI solution typically costs at least USD \$500 per month per user.

- While off-the-shelf BI tools are available, the learning curve of such tools for non-technical business users is often steep.

- Hosting a BI solution requires the support of a non-trivial, and often costly, hardware infrastructure.

- While there are a wide range of BI solutions available, SMEs often lack in-depth knowledge of BI to select the most appropriate solution for addressing the business's needs.

- Generating BI is often a non-trivial task which requires good knowledge and understanding of database modelling and data warehousing. Such technical know-how is often not readily available within most SMEs.

In summary, the challenges can be narrowed down to two main factors: cost involved and lack of knowledge among the available resource pool to handle complicated BI tools. While these factors are legitimate barriers for SMEs to adopting a BI solution, they should not prove detrimental in promoting the implementation of BI solutions in SMEs. To overcome the budget issue, there is a need to identify low cost BI solutions. For example, a standard BI solution is shipped as part of Microsoft's flagship product. Companies owning an MSDN licence can have access to Microsoft's 
standard BI solution at no extra cost. However, many SMEs may not even be aware of such an opportunity nor have the expertise required to start utilising it.

\subsection{Benefits of Adopting BI in SMEs}

According to a research by Scholz et al. (2010), there are lots of benefits SMEs could realise by using BI tools. Some of the most important benefits identified in the research include:

- Improvements in Data Support through providing easy access to data. Improved data validation and cleansing process also leads to reports generated using high-quality data.

- Improvements in Decision Support through providing rich visuals to support an accelerated decision-making process. In addition, BI tools could help identify risks and rectify them in a timely manner.

- Cost and Time Saving can be achieved by various BI tools such as dashboards and scorecards because the retrieval of the data will be faster and it is easier to interpret the visual output of business data analysis.

The cost involved could be subsidised by utilising technologies that are already part of the existing company infrastructure. For example, if a company adopts the Microsoft suite of business products, no extra cost is required for purchasing standard BI components. Furthermore, the cost for purchasing data visualisation tools can be reduced when lightweight web technologies are used to present the business intelligence. This, not only minimises the overall cost, it also removes the need for extensive training.

\section{BI IMPLEMENTATION IN AN SME}

\subsection{About the Company}

The current Business Intelligence project is carried out for a UK based company known as AGGORA Group which is specialised in catering equipment solutions (AGGORA Group, 2016). Their core business is in sales and service of catering equipment and they perform asset management for their clients who range from major corporates to small businesses. The company has approximately 150 staff. The company turnover is approximately $£ 25 \mathrm{~m}$ a year.
AGGORA Group wanted to improve their ability to manage, analyse and utilise data. They also wanted to have a data management system that is flexible and can adapt to future changes so that they can manage their business effectively and efficiently. Though the existing IT team had capabilities to generate reports for the management team to analyse, the report generation was a laborious and inflexible task and it slowed down the processing of company's core IT system because the database was not designed to facilitate report generation. Furthermore, as the company has been in business for over ten years, there were also some legacy IT issues that affected the efficiency of report generation. The leadership team has therefore decided to implement a BI solution to support their expansion plans for the next decade. However, the key challenge for implementing a BI solution for AGGORA Group was that they did not possess sufficient expertise in BI technologies. BI technologies such as data warehousing need advanced knowledge in database design and it is a demanding task. Furthermore, the IT staff within the company were already tied up to the day-to-day support and enhancement of the existing IT infrastructure. Hence, retraining existing staff was not a possible option.

The company secured funding from the UK government through the Knowledge Transfer Partnerships (KTP) scheme (InnovateUK, 2016) to investigate possible solutions for their business challenges. This enables an IT graduate to be employed and work on implementing a BI solution with guidance from a UK university. The IT graduate is employed by the university, but is based in the company and hence able to interact with the company employees daily. This enables all project stakeholders to be involved in the project development, with all views and concerns considered and addressed, from the beginning of the project. This helped to ensure a smooth roll out of the BI solution.

\subsection{Project Objectives}

The main source of the company's business data is generated by an in-house software developed for managing field services. This BI project focuses on:

- Analysing Client KPIs: Since the major business of the company is servicing catering equipment, it is important to understand whether the Service Level Agreements (SLAs) are met, whether the customers are satisfied, etc. 
- Analysing Engineers' Performance and Productivity: The company employs over 80 field service engineers. To better manage the engineers' time and the quality of their work, the company would like to measure and analyse the performance and the productivity of the engineers.

- Analysing the Financial Figures: As the growth rate of the company increases, analysing the costs and sales figures will enable the users to establish the economic state of play of the company.

- Asset Management: The company holds large volumes of historical information about the clients' equipment such as service history and overall spend. The ability to utilise this information will enhance the service provided to the clients.

In terms of maintainability and sustainability, as this is a KTP project and each KTP project is required to include a detailed plan for embedding new skills and knowledge into the company. Our project plan therefore has included provision for knowledge transfer and embedding through workshops and training.

\subsection{Implementation Methodology}

There are different approaches for implementing a BI solution. This could vary significantly based on the size and the IT budget of the company, as well as the company's knowledge in the BI field. For example, larger organisations with ample budget may purchase one of the several BI solutions available from a leading vendor. Such a BI solution may include staff training and supports. Such an organisation can also afford to employ a dedicated team to implement and support the system. However, SMEs are unlikely to be able to afford a high profile BI system bundle and the capabilities of such solutions are likely to exceed the needs of an average SME.

We have adopted an iterative, five-stage implementation process as advocated by McGonagle (2007):

- Planning

- Data Collection

- Data Analysis

- Data Distribution

- Feedback

\subsubsection{Planning}

Our BI implementation project starts with the planning phase. In this phase, the management team that would be consuming the intelligence articulate their requirements and establish the KPIs required to be measured. This forms the foundation for the subsequent phases.

Based on the objectives stated in Section 3.2, SQL Server Management Studio (SSMS) and SQL Server Data Tools (SSDT) were used for developing the required BI solution. In addition, the end user visualisation is achieved using Microsoft Power BI to generate self-servicing reports while the SQL Server Reporting Services (SSRS) was used to generate standard reports.

This decision was based on the existing IT infrastructure available in the company. Since the company already uses Microsoft SQL Server 2012 as its backend database, the Microsoft BI suite was a convenient option to implement this BI project. Microsoft SQL Server 2012 has a database system and other components needed for BI implementation.

SQL Server 2012 (Microsoft, 2016b) includes five core server components providing services ranging from data storage, management and security to specialist tools for data analysis, e.g. OLAP, and data mining. Apart from the Analysis Service, we have used the other four core components in this iteration.

\subsubsection{Data Collection}

Once the requirements are gathered and objectives are set, we then source the data and transform it into a form that will provide the required intelligence to the users. It is important to identify the data available in the source that can meet the project objectives.

As mentioned earlier, the source business data is generated by an in-house field service management system, the data is stored in the form of a relational database using the SQL server. The database stores information such as equipment management, cost and sales and engineers' timesheet information.

Based on the project objectives, it was decided that the data from the source would be transformed and stored into a data warehouse. One of the most commonly used technique for designing a data warehouse is dimensional modelling (Kimball and Ross, 2013). The main reasons for its popularity are that it results in a database schema which supports fast query performance and allows presentation of data in a user consumable format.

Though the dimensional model holds the same data as the source database, the dimensional model 
deals with un-normalised tables for extracting summarised and aggregated data. The main building blocks of building a dimensional model are: facts and dimensions. We have followed Kimball's four steps to dimensional modelling (Kimball and Ross, 2013):

1. Select the business process.

2. Declare the grain.

3. Identify the dimensions.

4. Identify the facts.

As per Kimball, a data warehouse could be designed based on the two kinds of schemata:

- Star Schema usually consists of fact tables linked to dimension tables using primary/foreign key relationships.

- Snowflake Schema consists of hierarchical relationships in a dimension table, with normalised, low-cardinality attributes appearing as secondary tables connected to the base dimension table by an attribute key.

For the purpose of this project, we have opted for a hybrid approach which is a combination of both star and snowflake schema. As we have used an iterative approach to build the data warehouse, some of the iterations utilised a star schema and others followed the snowflake schema. In order to maintain the data consistency among the shared dimensions, we have used the hybrid approach. The trade-off for utilising a hybrid approach is that the performance of the resulting data warehousing is slightly lower. However, due to the nature of our data, the slight degradation in performance was not noticeable by end users.

\subsubsection{Data Analysis}

Once the data warehouse is designed, it is populated with data from the live transaction database. This is known as an ETL (Extract-Transform-Load) process. Our ETL process uses Microsoft SQL Server Data Tools (SSDT) which provides a graphical interface that eases the design and implementation of data transformation and integration steps. As discussed earlier, our source data is from a single source, the ETL process involves mapping the data from the live transaction database to the data warehouse. The transformations performed in our ETL process include (Lacko, 2009):

- An Aggregate function which applies aggregation (minimum, maximum, average, sum, etc.) on the incoming set of data.

- A Conditional Split function which divides the set of data into more subsets.
- A Copy Column function which adds new columns, which are the copies of columns from input data set.

- A Data Conversion function which enables changing the data type of a column during the transformation.

- A Derived Column function which creates new columns derived from the values in the input columns.

Power BI (Microsoft, 2015) provides business users with useful data analysis and rich visualisation to facilitate informed decision-making. The desktop version of Power BI provides the ability to create powerful data connection, models and reports for data analysis. In this project, we have used Power BI desktop version to connect to the data warehouse. Once we have connected to the data source, we can adjust the data to meet our needs. We did some transformations using query editor in Power BI such as renaming column headers and changing the text to numbers. It is important to note that, the original data source is not affected; only this particular view of the data is modified. We have also created new measures using Data Analysis Expression (DAX) queries (Microsoft, 2016a) to provide aggregated data.

\subsubsection{Data Dissemination}

The output of the data analysis process is presented as business intelligence to the end users. We utilised the cloud-based version of Power BI to disseminate the results of data analysis to company managers. Users can quickly generate and deploy reports and dashboards and share them with other users.

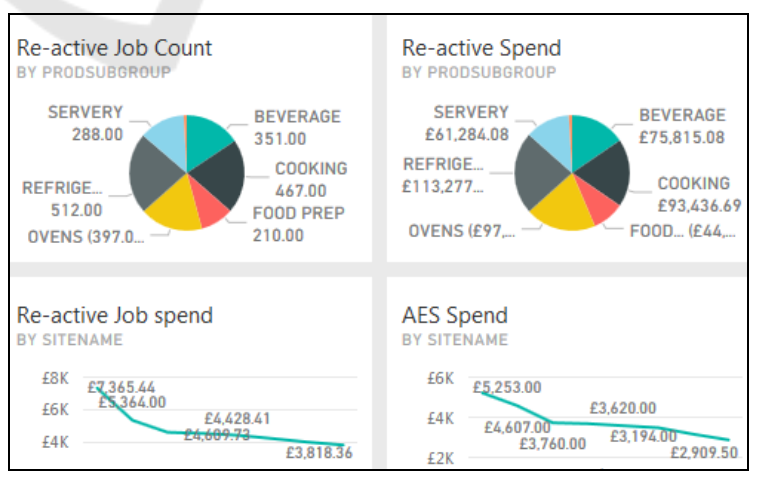

Figure 2: Sample reports created in Power BI.

Any Excel user who is familiar with using pivot tables and charts could be potential users of Power BI because the learning curve is not steep. With some basic training, we found that the end users were generally able to generate required business 
intelligence using Power BI without much technical assistance. Our Power BI users can edit their own reports, but they cannot edit the underlying data. This helps ensure data integrity.

We have chosen Power BI over the other selfservice BI options available in the market mainly because Power BI is fairly easy to use even for non IT experts and it has the ability to integrate with Office 365 (Microsoft, 2016d). Figure 2 shows some of our sample reports and dashboards created using Power BI. To ensure confidentiality, the clients' name have been removed in the sample reports.

Apart from Power BI, Microsoft SQL Server Reporting Services (SSRS) was used to create standard reports that could be delivered to relevant users on a regular basis. SSRS is a server based report generating software that comes as part of the Microsoft SQL Server suite. The SSRS provides a unique user interface based on Visual Studio that enables developers to connect to the relevant data (Microsoft, 2016e).

In this project, we used SSRS to create some standard KPI reports such as Engineer Performance and Productivity. These reports are embedded into an email which is sent to the respective managers for their analysis.

\subsubsection{Feedbacks}

At the end of each implementation iteration, feedback is collected from the end users. This feedback is used to inform the next iteration of implementation.

In this project, the feedback we obtained is from a steering group consisting of managers from various levels in the company who are also users of our BI solution. Our steering group members include commercial and technical directors as well as business operation managers. The steering group verifies the quality of the data and the accuracy of the intelligence provided and also provides feedback on how the solution can be improved in the future.

\section{BENEFITS}

Our experience shows that adopting BI has been beneficial to the company. The key beneficiaries so far have been the top and middle level management as well as the IT department. Since the introduction of the BI solution, the management gained the freedom to explore business information without requiring IT support and the IT team has been freed up to improve the quality and the granularity of information available. As a result, the management is able to make more informed business decisions as they have a clearer picture of the state of the business.

We have also carried out tangible benefit analysis of implementing a BI solution in the company. This analysis revealed that there were clear tangible benefits within six months of implementing the first iteration of the BI solution. The self-servicing reporting solution using Power BI has enabled top and middle level management to explore the business data and create their own ondemand BI reports without reliance on the IT department. This has led to a significant amount of time saving in the IT department as there is no longer the need for them to produce reports using queries and pivot tables in an ad hoc manner. This has also opened up more opportunities for the IT department to widen the scope of information available. The use of a specialised data warehouse has also reduced the processing load of the live database, making the core business system more efficient, reliable and effective.

The following table lists some of the key benefits identified so far:

Table 1: Tangible Benefits.

\begin{tabular}{|c|c|c|}
\hline 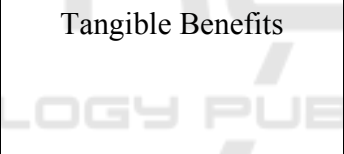 & $\begin{array}{l}\text { Value } \\
\text { Achieved } \\
\text { within } 6 \\
\text { months (£) }\end{array}$ & $\begin{array}{c}\text { Projected } \\
\text { Value within } \\
12 \text { months }(£)\end{array}$ \\
\hline $\begin{array}{l}\text { Cost saving resulted from } \\
\text { the introduction of Power } \\
\text { BI for self-service } \\
\text { reporting }\end{array}$ & $£ 4,202$ & $£ 8,404$ \\
\hline $\begin{array}{l}\text { Increased efficiency, } \\
\text { productivity and revenue } \\
\text { through new reports } \\
\text { generated using data } \\
\text { warehouse and Power BI }\end{array}$ & $£ 18,060$ & $£ 43,000$ \\
\hline $\begin{array}{l}\text { Time saving achieved for } \\
\text { IT team by using data } \\
\text { warehouse for generating } \\
\text { report }\end{array}$ & $£ 7,000$ & $£ 14,000$ \\
\hline Total: & $£ 29,262$ & $£ 65,404$ \\
\hline
\end{tabular}

\section{DISCUSSION}

In this section, we will summarise our experiences in implementing the first iteration of a BI solution within a UK SME. As a first remark, it is important to understand that implementing a BI solution is an iterative process and it is not trivial. Thorough 
understanding of the existing systems is required. The objectives and KPIs of the business should be well-defined before any implementation commences. In addition, this requires technical knowledge of BI concepts such as data warehousing, the ETL process and available data visualisation tools.

In terms of implementation strategy, we have chosen to deliver BI in a top-down manner, with the first set of BI solutions rolled out to top-level managers. This allowed top-level management to better understand the benefits of BI, and hence be more supportive of a wider exploitation of BI within the company.

To keep the costs down and to make the solution more accessible to a wide range of end users, we have deliberately used only a limited set of BI tools. Based on our experience, the Microsoft suite of BI tools can be used to support all aspects of business intelligence required in this project. Microsoft also provides tools for more advanced business data analysis such as creating data cubes and mining data for prediction analysis.

It is vital for an IT solution to be user-friendly so that there will be more buy-in from end users. The SSRS tools used to generate standard reports require some technical skills to work efficiently with it. On the other hand, Power BI supports an intuitive process for creating on-demand reports and it provides rich data visualisations. Most Excel users will be able to work efficiently with Power BI without much training. Furthermore, there are several tutorials available for demonstrating how to use Microsoft BI tools.

Finally, our experience shows that:

- BI solutions empower SMEs to understand their current performance and evaluate it against the company's KPIs.

- While many SMEs understand the benefits of implementing BI solutions, they are often put off by the seemingly complex and expensive implementation process. Our experience shows that affordable and relatively simple BI solutions exist and they can easily fit in SMEs' existing IT solutions.

- As BI solutions become more readily available, SMEs should possess better understand of their existing IT infrastructure before making any new investments.

- Implementing a BI solution involves various phases. A good understanding of these phases is needed to bring about success.

- In order to generate more accurate business intelligence, it is important to have high-quality data. This can be achieved using various transformation and cleansing steps in the ETL process.

- To facilitate efficient data retrieval and analysis, data warehouses cannot be created by simply joining tables from the live transaction database. It should be properly designed by following guidelines on dimensional modelling.

- The Microsoft suite of BI tools could be a perfect candidate for SMEs wanting to implement BI solutions, especially when they are already using other Microsoft business products.

- While technical barriers do exist, there are government schemes, in the case of UK and Europe, available to help SMEs to overcome such barriers through funding, recruitment and knowledge transfer.

\section{CONCLUSION}

BI tools provide analytic data and key performance information which enables organisations of all sizes to be managed efficiently. The usage of BI tools in SMEs was generally lower than that in large organisations. Studies show that SMEs appreciate the importance of streamlining their information resource to help them make important business decisions. They are also aware of the availability of many BI tools in the market. However, the technical expertise required to select the right combination of tools is not readily available in many SMEs. Furthermore, the general lack of sufficient IT budget prevents SMEs from investing time and money in adopting BI solutions.

In light of a BI implementation project, we have described how an SME can reap the benefits of BI using the Microsoft BI suite: Microsoft SQL Server for data modelling and Power BI for data analysis and visualisation. Based on our experience, we have also made a recommendation of how SMEs may approach implementing a BI solution, highlighting the need for good understanding of the existing data source, cleansing and transforming the data, creating a data warehouse based on Kimball's approach for storing the transformed data and presenting the data to the end users using various visualisation tools. A possible limitation of our work is that it is based on a company which has already adopted a Microsoftbased IT infrastructure and has accumulated a significant amount of business transaction data. We recognise that if our approach were to be applied in a company with a different IT infrastructure, the 
costs, benefits and time scales could be different. However, with Microsoft being the market leader in both operational database management systems and BI and analytics platforms (Gartner, 2015; 2016), our findings are relevant to a large number of businesses.

With the advancement of data mining and big data analysis, there are a wide range of algorithms and tools available for generating advanced business intelligence. This paper describes our first step in implementing a BI solution to an SME in the UK. Our next step includes using BI to: (1) achieve further potential enhancements within the organisation, (2) analyse the productivity of subteams within the enterprise and (3) perform margin analysis or implementing a more sophisticated account statement model. With the option available to integrate R scripts with Power BI, we also plan to investigate using predictive time series analysis or other advanced data analysis to provide forecasts.

\section{ACKNOWLEDGEMENT}

This project is supported by Innovate UK under the Knowledge Transfer Partnership (KTP) scheme [KTP number KTP009714].

\section{REFERENCES}

AGGORA Group. (2016). AGGORA, http://aggora.co.uk/ [accessed 15 August 2016].

Burstein, F. and Holsapple, C. (2008). Handbook on decision support systems. Berlin: Springer.

Chugh, R and Grandhi, S. (2013). Why Business Intelligence? Significance of Business Intelligence tools and integrating BI governance with corporate governance', International Journal of E-Entrepreneurship and Innovation, vol. 4, no.2, pp. 1-14. http://www.igi-global.com/article/why-businessintelligence/ 89282

European Commission (2016). What is an SME?, http://ec.europa.eu/growth/smes/business-friendlyenvironment/sme-definition/index en.htm [accessed 1 June 2016].

Fink, K. and Ploder, C. (2009). Knowledge Management Toolkit for SMEs. International Journal of Knowledge Management, 5(1), pp.46-60.

Gartner Inc. (2012). Gartner Executive Programs' Worldwide Survey of More Than 2,300 CIOs Shows Flat IT Budgets in 2012, but IT Organizations Must Deliver on Multiple Priorities, http://www.gartner. com/newsroom/id/1897514 [accessed 25 May 2016].

Gartner Inc. (2015). Magic Quadrant for Operational Database Management Systems, https://www.gartner.
com/doc/reprints?id=1-2PMFPEN\&ct=151013

[accessed 15 August 2016].

Gartner Inc. (2016). Magic Quadrant for Business Intelligence and Analytics Platforms, https://www. gartner.com/doc/reprints?id=1-2XXET8P\&ct $=160204$ [accessed 15 August 2016].

Google (2016). Google Charts, https://developers.google. com/chart/ [accessed 2 June 2016].

Horakova, M. and Skalska, H. (2013). Business Intelligence and Implementation in a Small Enterprise. Journal of Systems Integration, 4.2, pp.50-61.

Howson, C. (2008). Successful business intelligence. New York: McGraw-Hill

InnovateUK (2016), Knowledge Transfer Partnerships, https://connect.innovateuk.org/web/ktp [accessed 10 June 2016].

Kimball, R. and Ross, M. (2013). The Data Warehouse Toolkit: The Definitive Guide to Dimensional Modeling, $3^{\text {rd }}$ edition. Indianapolis, IN: Wiley.

Lacko, L. (2009). Business Intelligence v SQL Serveru 2008. Brno: Computer Press.

Luhn, H.P. (1958). A Business Intelligence System, IBM Journal of Research and Development, 2(4), 314-319.

McCabe, L. (2012). Closing the Business Intelligence Gap for Small Businesses, https://lauriemccabe.com/2012/ 01/27/closing-the-business-intelligence-gap-for-smallbusinesses/ [accessed 14 June 2016].

McGonagle, J. (2007). An Examination of the 'Classic CI Model. Journal of Competitive Intelligence and Management, 4(2), pp.71-86.

Microsoft. (2015). Frequently asked questions about Power BI Microsoft Power BI, https://powerbi. microsoft.com/en-us/documentation/powerbifrequently-asked-questions/?CorrelationId $=\mathrm{a} 0 \mathrm{~b} 3 \mathrm{a} 797$ a620-4e09-b346-1141a5f1d03d\&ui=en-US\&rs=enUS\&ad=US [accessed 27 May 2016].

Microsoft. (2016a). DAX Queries, https://msdn.microsoft. com/en-us/library/gg492201.aspx [accessed 13 June 2016].

Microsoft. (2016b). Editions and Components of SQL Server 2012, https://technet.microsoft.com/en-us/libra $\mathrm{ry} / \mathrm{ms} 144275 \% 28 \mathrm{v}=$ sql.110\%29.aspx [accessed 25 May 2016].

Microsoft. (2016c). Microsoft Developer Network (MSDN), https://msdn.microsoft.com/ [accessed 15 August 2016]

Microsoft. (2016d). Office 365, https://products.office. com/en-gb/business/explore-office-365-for-business [accessed 15 August 2016].

Microsoft. (2016e). Reporting Services (SSRS), https://msdn.microsoft.com/enus/library/ms159106.aspx [accessed 27 May 2016].

Negash, S. and Gray, P. (2003) Business intelligence, Proceedings of the Ninth Americas Conference on Information Systems (AMCIS 2003), Tama, Florida, pp. 3190-3199.

Papazoglou, M. P. (2003). Service-oriented computing: concepts, characteristics and directions, Proceedings of the Fourth International Conference on Web Information Systems Engineering, 2003 (WISE 2003), 
Rome, pp. 3-12. doi: 10.1109/WISE.2003.1254461.

Scholz, P., Schieder, C., Kurze, C., Gluchowski, P. and Böhringer, M. (2010). Benefits and challenges of business intelligence adoption in small and mediumsized enterprises. 18th European Conference on Information Systems.

Tutunea, M.F. and Rus, R.V. (2012). Business intelligence solutions for SME's. Procedia Economics and Finance, 3, pp.865-870.

Watson, H. and Wixom, B. (2007). The Current State of Business Intelligence, Computer, 40(9), pp.96-99. 